Revue d'histoire de l'Amérique française

REVUE D.HISTOIRE DE L'AMÉRIQUE FRANÇAISE

\title{
Le voyage d'Alexandre Vattemare au Canada : 1840-1841
}

\section{Elisabeth Revai}

Volume 22, numéro 2, septembre 1968

URI : https://id.erudit.org/iderudit/302782ar

DOI : https://doi.org/10.7202/302782ar

Aller au sommaire du numéro

Éditeur(s)

Institut d'histoire de l'Amérique française

ISSN

0035-2357 (imprimé)

1492-1383 (numérique)

Découvrir la revue

Citer cet article

Revai, E. (1968). Le voyage d'Alexandre Vattemare au Canada : 1840-1841.

Revue d'histoire de l'Amérique française, 22(2), 257-299.

https://doi.org/10.7202/302782ar d'utilisation que vous pouvez consulter en ligne.

https://apropos.erudit.org/fr/usagers/politique-dutilisation/ 


\section{LE VOYAGE D'ALEXANDRE VATTEMARE AU CANADA: $1840-1841$}

Un aperçu des relations culturelles franco-canadiennes: 1840-1857*

De nos jours, le nom d'Alexandre Vattemare est presque complètement tombé dans l'oubli. ${ }^{1}$ Pourtant, au siècle dernier, pendant plusieurs décennies, il a joui d'une popularité prodigieuse dans presque tous les pays d'Europe, ainsi que dans les grandes villes de l'Amérique du Nord, y compris le Canada. Sur ce continent, grâce au rôle prépondérant qu'il a joué dans la fondation de la Bibliothèque publique de Boston et aux efforts faits pour en créer d'autres, son souvenir est resté plus vivant.

Pour plus de clarté nous allons tracer le plan de cette étude:

I. Le voyage d'Alexandre Vattemare au Canada: 1840-1841

1. Biographie d'Alexandre Vattemare

2. Le voyage au Canada

II. Faribault

1. Relations entre Faribault et Vattemare

2. Relations entre Faribault et $\mathrm{A}$. de Puibusque.

\section{1 - Biographie d'Alexandre Vattemare}

Né à Paris le 8 novembre 1796, mort dans la même ville le 7 avril 1864, Alexandre Vattemare a commencé sa carrière vertigineuse dans le tumulte des révolutions et des guerres napoléoniennes. Son père était avocat, provenant d'une famille normande issue de Jean de Vattemare, seigneur de Vascovy, ${ }^{2}$ anobli

* Extrait d'un ouvrage en préparation.

1 Voir bibliographie à la fin de cet article.

$2 \mathrm{H}$. Vattemare, "Notices of the life of A. Vattemare", Hist. Mag. (1868), IV: 297. 
par Louis XI. ${ }^{3.4}$ Dès le début de la révolution de 1789 , il jugea plus prudent de se retirer avec sa famille dans sa propriété de Lisieux en Normandie où les talents particuliers d'Alexandre eurent tôt fait de se révéler. ${ }^{5}$ En effet, c'est là, entre l'âge de sept à onze ans, que le jeune garçon commença à se servir de ses dons de ventriloque, causant beaucoup d'ennuis aux villageois à qui il jouait des tours pendables. ${ }^{6}$

En dépit des remontrances sérieuses de son père, Alexandre ne put s'empêcher d'exercer ses talents. C'est pour cette raison qu'il se fit renvoyer du séminaire de Lisieux où il était entré pour se conformer au désir de sa mère. Il subit le même sort à l'hôpital St-Louis de Paris où pourtant de brillantes études lui avaient valu un poste d'aide-chirurgien après un stage de quelques mois seulement. ${ }^{7}$

Cependant, les événements historiques amenèrent une nouvelle modification dans la carrière d'Alexandre. On est en 1814, en pleine campagne de France, les épidémies ravagent Paris. Obligé d'avoir recours à toutes les forces disponibles, l'hôpital de la Pitié engage le jeune médecin sans diplôme. Alexandre soigne la population civile et militaire et, par sa gentillesse et sa bonne humeur, se fait apprécier de ses patients. Aussi, lorsqu'il s'agit de rapatrier en Prusse un groupe de trois cents militaires de l'armée des Alliés, ceux-ci demandent-ils que ce soit le jeune officier de santé qui les raccompagne. ${ }^{8}$ Alexandre accepte - et cette circonstance décide de son destin. En effet, la période des Cents Jours le trouve bloqué à Berlin devant l'alternative d'entrer dans l'armée alliée ou de rester en Prusse comme prisonnier de guerre. Vattemare choisit la deuxième possibilité. Sans res-

\footnotetext{
3 Ecusson de la famille Vattemare, 1666. B.N. Ms. Document inédit.

4 Contrat entre Dame Judith Leblanc, veuve de Josias de Vattemare, escuyer, et M. Romain Varin, 1720. B.N. Ms. Doc. inéd. 15.

5 Memoirs and anecdotes of Monsieur Alexandre... (London, 1822),

6 J. L. Dargent, “Al. Vattemare”, Bull. des Bibl. de Fr., (1964), 24.

7 E. M. Richards, "A.V. and his System of international exchanges", Medical Ass. Bull., (1944), 415.

8 Pour cette mission délicate, accomplie avec zèle, il sera décoré par la Croix de fer prussienne. H. Vattemare, op. cit., 298.
} 
sources, n'ayant pas le droit d'exercer la médecine, il lui faut se trouver des moyens de subsister. Sur les conseils de l'ambassadeur de France, M. de Caraman, ${ }^{\circ}$ il décide de faire appel à ses talents de mime et de ventriloque qui jusqu'à présent ne lui avaient valu que des ennuis.

Le profit de sa première représentation devait aller à une famille d'émigrés français ruinés par la révolution. Âgé seulement de 17 ans, il prit à sa charge cette famille de quatre membres, les Thabouis de Guidon, et pendant trois ans fit le tour de l'Europe avec eux. Ayant épousé Mlle Thabouis de Guidon, il accumula succès sur succès avec des pièces écrites à son intention où, sous le nom de M. Alexandre, il jouait jusqu'à quarante différents rôles en une soirée. ${ }^{10}$

M. Alexandre était né et devait briller à travers l'Europe pendant un quart de siècle, fêté et adulé par trois empereurs et plusieurs rois. ${ }^{11}$ Il joue dans la plupart des capitales, et ce, dans la langue du pays: allemande en Allemagne et en Autriche, anglaise sur l'île britannique, française en France, dans les Pays-Bas, en Pologne, Russie, Hongrie et Italie ${ }^{12}$. Ses talents de mime et de ventriloque ont été, par la suite, reconnus par les dictionnaires professionnels ${ }^{13}$ qui le citent parmi les meilleurs de la profession. ${ }^{14}$

Les plus grands noms de la littérature et du monde des arts de son époque apprécient son talent. Goethe lui offre quelques lignes élogieuses, signées le 8 juin $1818 ;^{15}$ Walter Scott lui dédie tout un poème intitulé: Lines addressed to Monsieur Alexandre,

${ }^{9}$ "Strange career of an artist", Hours at home (1858), 535.

$10 \mathrm{Wm}$. B. Trask, "[Biographical sketch of A.V.], N. Engl. hist. \& geneal. Register (1865), 367.

11 J. P. Quincy, "A.V.: his services in connection with the Boston City library", Mass. hist. soc. Proceedings, 1884-1885, 265.

12 J.-L. Dargent, op. cit., 334.

13 Henry Lyonnet, Dictionnaire des comédiens français ... (1904), 15.

14 John Chambers, "Ventriloquism", Mirror of literature, amusement and instruction, 28: 355-356.

15 "Strange career of an Artist", 535. 
the celebrated ventriloquist, $1824^{16}$, pour ne citer que les plus célèbres. Car durant ses pérégrinations, il eut l'heureuse idée de collectionner des autographes, poèmes et dessins des illustres personnages qu'il avait rencontrés et qu'il publia en 1839 sous le titre d'Album cosmopolite ${ }^{17}$. Parmi les participants de l'Album figurent des noms aussi hétéroclites que ceux des divers souverains, ambassadeurs, hommes de lettres, etc. de son époque.

Il va sans dire que le métier d'occasion choisi dans un moment de détresse ne tarda pas à procurer à Vattemare des satisfactions pécuniaires. Rien qu'à Saint-Pétersbourg ses représentations lui rapportent 100.000 roubles, l'équivalent de 50,000 dollars environ. Grand seigneur, M. Alexandre offre la moitié de cette somme au bénéfice des villages russes récemment incendiés. Il répétera ce geste à Dublin où il met 5,000 dollars à la disposition des œuvres de charité irlandaises. ${ }^{18}$

Cet esprit philanthropique le conduit naturellement vers la seconde étape de sa carrière où il se distinguera comme promoteur d'échanges culturels et fondateur de bibliothèques. C'est lors de ses nombreux voyages à travers l'Europe que lui vient l'idée de son système d'échange. Artiste consciencieux, il étudie les richesses nationales dans les musées et bibliothèques afin de se créer des costumes et des décors convenant à chaque pays. Il s'apercevra bientôt que les collections de documents, de médailles, de monnaies de ces institutions contiennent beaucoup de doubles tout en présentant aussi un bon nombre de lacunes. Petit à petit il entreprendra d'organiser des échanges sur un plan bénévole, simplement pour rendre service aux diverses institutions. C'est à partir de 1832 qu'il commence à élaborer un système d'échanges entre la France et la Bavière, influencé par une lettre de M. Lichtenthaler, directeur de la Bibliothèque royale de Munich. ${ }^{19}$

${ }^{16}$ Sir Walter Scott, The poetical works, 732, Cité dans: E. M. Richards, A. Vattemare, Thèse (1934).

17 A. Vattemare, Album cosmopolite ..., 1837-1840.

18 H.-A: Dubuque, A.V̈., fondateur de la bibl. de Boston, 97-100.

19 César Moreau, le Correspondant, 1848, 1849, dans: A. Passier, Les Echanges intern. litt. et scient. (1880), 11-27. 
Enthousiasmé par ses premiers succès, il fonde à Paris, par ses propres moyens, la Société européenne des échanges qui rend des services appréciables. Mais ce qui fut adopté aisément en Autriche, en Prusse, en Angleterre, ne l'est pas en France. Vattemare a beau présenter des pétitions aux deux Chambres en 1836 et en 1839, il a beau démontrer que de précieux doubles se perdent ainsi pour la France, il ne réussit pas à obtenir une subvention du gouvernement français. Finalement, cédant aux instigations de la Fayette, Lamartine, et du général Cass, ambassadeur des États-Unis en France, il décide d'étendre son champ d'action au Nouveau-Monde où les possibilités sont plus grandes. $^{20}$ Il part donc en 1839 pour un voyage de trois mois qui va en durer dix-neuf. Quant à son succès, citons l'un de ses commentateurs, César Moreau, selon qui "les voyages de M. Vattemare en Amérique sont les plus extraordinaires, les plus prodigieux, les plus incroyables qui aient jamais été accomplis" ${ }^{21}$.

C'est au cours du premier voyage de Vattemare que se situe sa visite au Canada qui fera l'objet de la deuxième partie de cette étude. Ici, nous nous bornerons à parler des résultats obtenus aux Etats-Unis qui dépassent probablement tout ce qu'il avait pu espérer.

Avant d'entrer dans les détails de ses activités, il serait curieux de noter que, profitant de ses expériences d'Europe, il va se servir des talents de l'artiste pour frayer le chemin au philanthrope. Comme il aimait à le répéter: les portes que ne savait pas ouvrir M. Vattemare, M. Alexandre les lui ouvrait. ${ }^{22}$ Il fera donc ses débuts dans la vie publique par une représentation au Park Theatre de New York, le 28 octobre 1839.23 Cependant les succès obtenus dans la propagation de son Système lui firent négliger ses intérêts financiers d'artiste. Du moins, c'est ce que lui fait entendre son associé, J. H. Mauvers, un artiste américain, dans une lettre remplie d'amertume et de

20 Ibid., 17.

21 Ibid., 18.

22 "Strange career of an Artist", 539.

23 E. M. Richards, A.V., Thèse (1934), 6. 
prédictions qui ne se réalisèrent que trop. Comme par la suite on a reproché de certains côtés à Vattemare d'avoir mêlé ses propres intérêts à ses buts philanthropiques, nous noterons certains passages de cette lettre qui témoignent des sacrifices que dut faire Vattemare pour ce qui allait devenir selon le ministre Guizot l'idée fixe de sa vie.

Selon l'auteur de la lettre, pour peu que Vattemare ait employé pour leurs intérêts communs la moitié seulement des efforts consacrés à son Système d'échanges, chacun d'eux aurait en ce moment 3,000 dollars de plus. Il essaie donc de rappeler Vattemare à son devoir et le prie instamment de placer ses propres intérêts et ceux de sa famille au-dessus de ceux de l'humanité, le mettant en garde contre les dangers que comportent les chimères du genre de son Système. Souvent les promoteurs de ces projets humanitaires y perdent et leur fortune et leur santé sans toutefois acquérir la reconnaissance qui leur serait due. ${ }^{24}$

Ainsi, aux États-Unis, comme le démontre ce témoignage émouvant d'un associé frustré, $M$. Alexandre cède le pas à $M$. Vattemare. Son but initial, la propagation de son Système d'échanges va bientôt bifurquer en un autre projet, celui de la fondation de bibliothèques et d'institutions culturelles qu'il jugeait insuffisantes comparée à celles de l'Europe. En somme, c'est sur ce territoire secondaire qu'il a réussi à obtenir les résultats les plus durables: "en 1853 il avait inscrit 130 bibliothèques et institutions à son crédit" ${ }^{25}$ dont la Bibliothèque publique de Boston où son nom, sur le marbre de la porte d'honneur, est commémoré en caractères de cuivre parmi ceux des autres fondateurs de la bibliothèque.

Évidemment, son prestige d'artiste, ses relations avec le grand monde dont témoignait son Album cosmopolite qu'il avait apporté en guise de lettre d'introduction, contribuèrent beaucoup

24 Ibid., 9.

25 Justin Winsor, Memorial History of Boston, IV: 286 n (cité dans H.-A. Dubuque, op. cit., 222). 
à son succès exceptionnel. ${ }^{26}$ Sa prestance devait aussi l'avantager. À la Bibliothèque publique de New York, parmi les lettres de Vattemare, on en garde une contenant une mèche de ses cheveux qu'il avait envoyée à une dame de la haute société de Baltimore, Mrs. C. Chase Oldfield, qui lui avait demandé cette faveur. ${ }^{27}$

Grâce aux qualités personnelles et à l'endurance physique exceptionnelle de Vattemare, son voyage se solde donc par des résultats considérables. Depuis son premier discours public au Clinton Hall de New York où il expose son Système des échanges internationaux, il ne compte que des succès: son mémoire sur le même sujet adressé au Congrès de Washington est accepté favorablement par la voie du comité de la bibliothèque. ${ }^{28}$ Vattemare continue son tour des états et dans plus de cinquante grandes villes du continent nord-américain - dont Boston, Montréal et Québec marquent les étapes les plus brillantes propage avec un succès fulgurant l'idée de son Système d'échanges et celle de la fondation de bibliothèques. ${ }^{29}$

À son départ de New York, il reçoit des signes de la reconnaissance officielle française - morale sinon pécuniaire - par la lettre du ministre de France à Washington, M. de Bacour, dans laquelle celui-ci, en termes chaleureux, accuse réception des "richesses scientifiques et bibliographiques que, par vos nombreuses démarches et influence personnelle, vous venez d'acquérir à la France. Je suis heureux de pouvoir vous féliciter de ce beau succès, aussi honorable pour vous, Monsieur, que pour la nation américaine, dont vous avez su réveiller les sympathies pour notre chère patrie." 30

26 E. M. Richards, A.V., thèse (1934), 97.

27 Alexandre Vattemare, Copy-press letter-book, No. 3. Lettre autographe, signée, datée de Washington, Aug. 16, 1848. En voici un extrait: "Madame, Answering to the highly flattering compliment you are bestowing upon the humble projector of the system of intellectual union of nations, by insisting upon having a lock of his hair, I beg you to accept the enclosed ...", Doc. inéd.

28 Alexandre Vattemare, Memorial of Alexandre Vattemare to the honorable [sic] the Senate and House of the U.S...., Dec. 10, 1839 (U.S., 26th Congress 1st Sess., House doc. no. 50), cité dans: E. M. Richards, $A . V$., thèse (1934).

29 E. M. Richards, A.V., thèse, 14-23.

30 A. Passier, op. cit., 23. 
Muni de ses dons et de mandats américains, Vattemare rentre en France avec un prestige considérablement accru. Comme le fait remarquer Moreau: "les encouragements qui lui avaient été prodigués en Amérique, les résolutions des assemblées populaires, les actes des législatures et du Congrès étaient des arguments qu'avec son esprit, si fertile en ressources, il n'était pas embarrassé de faire valoir. Il apportait d'ailleurs d'autres et non moins incontestables preuves du bon vouloir des gouvernements et des peuples de l'Union: "plus de douze cents volumes, des cartes géographiques, des herbiers et un échantillon de fer oxydulé des montagnes du Missouri pesant de 1,000 à 1,220 kilogrammes". ${ }^{31}$

Enthousiasmé par ces résultats, Vattemare réorganise son Agence européenne d'échanges, distribue les dons qu'il a reçus et se met en devoir de se procurer de nouveaux éléments d'échanges pour l'Amérique.

Son deuxième voyage, qui s'effectue en mai 1847, sur les deux bords de l'Atlantique, suscite beaucoup plus d'intérêt que le premier. Nous pouvons juger de l'opinion publique par la presse contemporaine, dont quelques coupures de journaux provenant des manuscrits de G.-B. Faribault - probablement un don de Vattemare à ce dernier - peuvent nous donner une certaine idée. ${ }^{32}$

L'une des coupures mentionne le voyage de Vattemare et "le précieux chargement confié au packet Admiral par cet apôtre persévérant de l'union intellectuelle de la France et de l'Amérique". L'autre article, ayant paru dans le Journal du Havre du 8 mai (1847), décrit "une certaine quantité de colis portant pour inscription: Échange international, et adressés à divers états de l'Union américaine. Ces colis, au nombre de cinquantedeux, contiennent effectivement une riche collection de 12,000

31 Ibid., 22-23.

32 Bibliothèque Nationale du Québec. MS. Collection G.-B. Faribault. Notes des livres à acheter. Calepin. 3 coupures de journaux: 1) M. Vattemare et les échanges internationaux; 2) MM. Vattemare et Leverrier, $1847 ; 3)$ A. Vattemare au Havre, le 8 mai [1847]. 
volumes, cartes et gravures, envoyée aux Etats-Unis par le roi, les ministres, plusieurs villes et corps savants de France, en retour d'un envoi de même nature précédemment expédié des Etats-Unis. Cet échange entre les deux pays, de leurs richesses intellectuelles ... est dû à l'initiative et à l'entremise d'un homme qui a fait de cette œuvre d'alliance littéraire le but de toute sa vie."

De l'autre côté de l'Atlantique, où Vattemare retourne après une absence de six ans, la réception n'est pas moins enthousiaste. D'ailleurs, Vattemare avait eu soin d'entretenir ses relations avec plusieurs états, dont New York, le Maine et le Massachusetts, ainsi qu'avec le Canada par sa correspondance avec GeorgesBarthélemy Faribault, que nous ferons connaître plus loin.

Arrivé à New York avec son chargement évalué à 80,000 dollars ${ }^{33}$, Vattemare entreprit une nouvelle campagne énergique. Un deuxième mémoire présenté au Congrès de Washington en février 1848 couronna ses efforts du succès tant souhaité. Le comité de la bibliothèque du Congrès nomma Vattemare à l'unanimité son agent officiel, chargé d'effectuer les échanges entre la France et les États-Unis. De plus, des bills spéciaux décrétèrent que tous les documents et objets transmis par cet agent seraient reçus dans les ports de l'Union franes et quittes de tous droits. $^{34}$

De retour à Paris en 1850 , Vattemare semble au faîte de sa carrière. Son agence, officiellement reconnue, fonctionne avec une certaine régularité, les échanges s'effectuent de part et d'autre. Cependant les faiblesses inhérentes au système se font bientôt sentir; les buts de l'organisation étaient trop divers pour qu'un seul homme, même avec l'aide de deux membres de sa famille, ait pu s'en acquitter. ${ }^{35}$ Comme le fait remarquer Passier: "M. Vattemare qui avait eu raison quant à la théorie, ne commit-il pas une erreur de pratique en voulant constituer une "Agence

33 E. M. Richards, A.V., thèse, p. 36. Notons que la valeur du dollar en 1847 correspondait environ au quintuple de celui de nos jours.

84 Ibid., 38-40.

35 Ibid., 48-54. 
Universelle des Échanges Internationaux ?" Nous sommes portés à le croire, car c'était une conception trop vaste pour être réalisable." 36

Sur ces entrefaites, plusieurs états retirent leur mandat, en raison des irrégularités qui se produisent dans le fonctionnement du système. ${ }^{37}$ Pour comble de malheur, les événements internationaux vont encore faire sentir leur choc dans cette carrière tumultueuse. D'abord c'est la guerre de Crimée (18541856) qui menace l'existence de l'Agence. Vattemare se plaint à son entourage d'être ruiné et d'avoir perdu 200.000 francs. ${ }^{38}$ Mais c'est finalement la guerre de Sécession des États-Unis (1860-1865) qui lui donne le coup de grâce. Tous les privilèges américains sont retirés, y compris les subventions. Beau joueur, Vattemare n'abandonne pas la lutte, comble les lacunes avec sa propre fortune. Sentant ses forces physiques diminuer, il fait un ultime effort et, le 28 février 1864, moribond, alité, dicte une lettre adressée à M. Duruy, ministre de l'Instruction publique, "d'où s'exhalent sans amertume, la crainte et le regret de voir sur le point d'être anéanti, faute de la protection du Gouvernement, un travail de Sisyphe qui avait duré trente et un ans". ${ }^{39}$

Dans cette lettre émouvante, Vattemare fait l'historique de son Agence. Il rappelle les chiffres cités par le ministre Guizot: "plus de trois cent mille volumes, un nombre considérable d'objets d'art, d'histoire naturelle et d'industrie, des milliers de graines et de boutures échangées entre la France et diverses nations des deux hémisphères, par les efforts isolés d'un seul homme sans nom et sans fortune, que ne serait-on pas en droit d'attendre de semblables relations, aussitôt qu'elles seraient placées officiellement sous l'égide du Gouvernement français et établies enfin sur une échelle en rapport avec leur importance?" 40

36 A. Passier, op. cit., 31.

37 E. M. Richards, A.V., thèse, 62-72.

38 Adolphe de Puibusque, Lettre à G.-B. Faribault, Paris, 21 juin, 1854, A.P.Q.

39 A. Passier, op. cit., 28.

40 Ibid., 29. 
Ce dernier appel, où pourtant Vattemare fait allusion à ses forces défaillantes, reste sans réponse. Meurtri, découragé, Vattemare s'éteint quelques semaines après. Son Agence, malgré les efforts de son fils Hippolyte et de son gendre, Célestin Moreau, s'anéantit avec lui.

La disparition de l'œuvre capitale de Vattemare ne signifie pas cependant l'échec de ses efforts. Ses idées, ses innovations ont survécu à leur créateur, ainsi que l'institution fondée pour les promouvoir. Les échanges internationaux ont continué, mais sur une base officielle, exécutés par les gouvernements mêmes. Ainsi, les idées qu'il avait propagées avec tant d'ardeur sont tombées, pour ainsi dire, dans le domaine public, et se sont réalisées dans toute leur ampleur, sans son entremise.

Il n'en reste pas moins, que les résultats obtenus de son vivant sont gigantesques et témoignent d'une capacité de travail, d'une endurance sans pareilles. Peut-être était-ce ce même don de Protée qui lui permettait de jouer sur la scène sept à huit personnages à la fois qui le rendait capable de faire de ces tours de force dans la vie. Aussi E. M. Richards ne peut-elle s'empêcher d'être impressionnée par son rendement: rien qu'aux États-Unis il a été en contact avec cent organisations, grâce auxquelles plusieurs milliers de livres et de documents furent échangés par son Agence. En considérant toutes les ramifications de son système, elle trouve étonnant que le Système ait pu si bien fonctionner. ${ }^{41}$

Quant aux insinuations mettant en doute la philanthropie de Vattemare, on pourrait leur donner une réponse significative par les faits mêmes. Vattemare s'est souvent plaint d'avoir sacrifié sa fortune personnelle pour les besoins de son Agence. Ces déclarations ont été confirmées par un document inédit que nous avons eu l'occasion de consulter. La succession de Vattemare, y compris une maison à Marly-le-Roi, a été évaluée à 156.546 francs et répartie entre sa veuve et ses cinq enfants. ${ }^{42}$

41 E. M. Richards, A.V., thèse, 106.

12 Paris. Direction générale des impôts. Déclaration de succession d'Alexandre Vattemare. 8 sept. 1864 . Outre le relevé de ses biens mobiliers 
Si l'on songe que du temps de M. Alexandre il avait facilement cédé la moitié de ses honoraires gagnés en Russie, 125.000 francs environ, correspondant à la presque totalité de sa succession, on peut se faire une idée des gains auxquels il avait renoncé pour pouvoir se consacrer à ses buts philanthropiques. D'ailleurs, il suffit de nous rappeler à cet égard la lettre prémonitrice déjà citée de l'artiste de New York, son associé (voir p. 262 de cette étude).

D'autre part, il ne fait pas de doute que les qualités personnelles de Vattemare ont été reconnues par la majorité de ses contemporains. L'un des témoignages les plus émouvants se trouve dans le discours de l'un de ses grands amis, Josiah Phillips Quincy, maire de Boston et président de l'Université Harvard, prononcé à la mémoire de Vattemare devant la Massachusetts Historical Society, en 1884. Après avoir énuméré les mérites de Vattemare, dans la fondation de la Bibliothèque publique de Boston et dans la promotion des relations culturelles entre leurs deux pays, il ajoute cette appréciation, dictée autant par son amitié que par son sentiment de justice:

Voilà une carrière remplie d'œuvres admirables qui donne raison à notre ministre en France, M. Cass, lorsqu'il dit qu'Alexandre Vattemare sera placé au rang des bienfaiteurs de l'humanité et, comme eux, il sera récompensé par l'estime universelle. ${ }^{43}$

\section{2 - Le voyage au Canada: octobre 1840 - février 1841}

$\mathrm{Au}$ Canada, la visite de Vattemare se déroule dans une atmosphère pour le moins aussi enthousiaste qu'aux Etats-Unis.

et immobiliers nous y retrouvons le nom de sa femme et de ses cinq enfants, ses héritiers: sa veuve, née Jeanne Thabouis de Guidon; ses enfants: 1) Hippolyte V. et son épouse, Pauline V., 2) Mme Pierre-Adolphe Muziau, dit Nancy, (Pauline V.), 3) Mme Célestin Moreau (Clémentine V.), 4) Alfred Marie Maurice V., prêtre, vicaire à l'Eglise de la Sainte-Trinité à Paris, 5) Mme Ignace Tyl (Zénaïde V.). Doc. inéd.

Aux Archives de la Seine et de la Ville de Paris, nous avons retrouvé d'autres documents inédits concernant la famille Vattemare: l'acte de mariage d'A.V., 3 actes de naissance et 4 actes de mariage de ses enfants. $1885,262$.

43 Josiah Ph. Quincy, "A.V....", Mass. hist. soc., Proceedings 1884- 
Sans doute, la situation politique à cette époque se prête-t-elle particulièrement bien à ce genre de manifestations nationales et culturelles. Il serait intéressant de citer à cet égard une source française de l'époque qui a songé à insister sur le climat politique de la visite:

Avant de se rendre dans l'Etat du Maine M. Vattemare avait voulu voir le Canada. Là une population française l'appelait comme à la Louisiane; il y arriva vers la fin d'octobre 1840. Les circonstances alors étaient difficiles. Les ferments de la dernière insurrection bouillonnaient encore. Il y avait entre les deux races des ressentiments déjà demi-séculaires qui s'étaient accrus par la victoire récente du gouvernement... M. Vattemare ne désespéra pourtant ni des Canadiens, ni de lui-même. Il continua d'avoir foi en son œuvre, et il fit bien. ${ }^{44}$

D'autre part, un Canadien, témoin de la visite, reflète les réactions chaleureuses locales. L' "apothicaire" Romuald Trudeau note dans son journal, intitulé "Mes tablettes", en date de janvier 1841:

Il est arrivé cet automne un voyageur philanthrope du nom d'A. Vattemare, dont le but est d'établir au Canada une institution d'un genre nouveau pour ce pays, mais dont il a déjà doté plusieurs pays étrangers où il a été favorablement accueilli, tel que la France, l'Angleterre, la Turquie, les Etats-Unis et autres. Cette noble institution dont Mr. Vattemare paraît avoir le mérite de l'invention et qu'il se fait gloire de propager par ses efforts et ses sacrifices dans tout le monde civilisé, tend à établir une espèce de commerce et de fraternité entre toutes les ramifications de la grande famille humaine, au moyen d'échange en fait d'objets d'art, de sciences, de littérature et des découvertes en tout genre... Toutes les démarches sont couronnées d'un plein succès; déjà le Conseil a passé une ordonnance à l'effet d'autoriser l'emprunt d'une somme 50.000, afin de pourvoir aux moyens de construire sans délai un édifice convenable et monumental pour y recevoir

14 A. Passier, op. cit., 27. 
les richesses du génie et de la science qui seront fournies par les diverses nations étrangères. ${ }^{45}$

Ainsi, selon cette note personnelle, Vattemare semble avoir suivi la même ligne de conduite qu'aux États-Unis: il se présente avec le but de propager son système d'échanges, mais, ne trouvant pas sur les lieux les institutions nécessaires, il essaie de persuader les autorités locales d'en créer pour les besoins de la cause. Comme à New York, il apparaît sous son double aspect de M. Vattemare - philanthrope et M. Alexandre - ventriloque, donnant des représentations pour le bénéfice de son institution.

La presse contemporaine prodigue des louanges effrénées tant au philanthrope qu'à l'artiste. Homme de théâtre, sachant apprécier la publicité, Vattemare s'est constitué un album de coupures. A la Bibliothèque publique de New York où ses écrits sont conservés dans neuf boîtes, la troisième renferme son "Scrapbook", spicilège où il a collé des coupures provenant de la presse de langue française et anglaise, chronique fidèle des événements mémorables de cette période de quatre mois et demi. ${ }^{46}$

Enthousiaste, le Fantasque lui consacre deux longs articles en première page. Le premier rend compte du Système et de l'Institut que propose "ce grand philanthrope, et homme sans pareil"; le deuxième exhorte les Québecois à se hâter de prendre une décision de peur de se laisser devancer par les Montréalais. Dans la chronique théâtrale, des entrefilets élogieux rapportent le succès des représentations en deux langues de l'artiste incomparable qu'est M. Alexandre. ${ }^{47}$

L'Aurore des Canadas comble de louanges celui qui était "l'ami des monarques, comme le favori de toutes les muses d'Europe" dans nombre d'articles et de comptes rendus qui suivent les événements de très près. Son arrivée est annoncée

45 Romuald Trudeau, Mes tablettes (janv. 1841), Mss, B.N.Q. de journaux.

46 Alexandre Vattemare, Copy-press letter-books, No. 3. Coupures

47 Ibid.. Le Fantasque, vol. 3, No 20 (8 févr. 1841), 117-148. 
comme celle du "plus grand et du plus zélé philanthrope qui ait peut-être jamais existé" ${ }^{48}$. Le journal publie, entre autres, la lettre du gouverneur lord Sydenham. Le patronage exceptionnel qu'il va accorder à cette cause s'explique par les termes chaleureux dont il le gratifie. Il commence par rappeler à Vattemare qu'il avait déjà eu l'occasion de lui témoigner son admiration en Europe; en effet, c'est lui qui avait présenté le projet de Vattemare aux Communes britanniques, en sa qualité de ministre du Commerce, sous son nom original de l'honorable Poulett Thomson. En soulignant le caractère humanitaire et universel du Système, capable de se servir "du terrain neutre des sciences et des arts pour faire taire les haines de race ou de parti", il termine sa lettre par ces mots:

Veuillez croire, monsieur, que mes vœux les plus sincères accompagnent vos efforts et que je serais flatté de pouvoir leur prêter mon faible appui. Votre triomphe sera celui de l'humanité.49

La presse de langue anglaise ne le cède en rien à celle de langue française, comme en témoignent les nombreuses coupures de la Montreal Gazette, du Montreal Herald, etc.50

En dehors de la chronique des événements de la visite, le Montreal Herald publie une longue lettre adressée au rédacteur, avec la signature de "A Reasoner" dont l'Aurore des Canadas reproduit une traduction complète signée par "Un Raisonneur". Apparemment, certaines divergences d'opinion avaient dû s'exprimer, car le lecteur juge bon d'analyser minutieusement les projets de Vattemare, tout en rassurant le public qu'il n'aura que des avantages à tirer de l'Institut auquel il aura libre accès. La lettre se termine par un parallèle entre M. Vattemare et $M$. Alexandre:

Nous pouvons bien dire de lui, "voir est croire". Il est contre son gré, obligé de paraître sur le théâtre comme un autre lui-même, le célèbre ventriloque Alexandre, et quoique ce ne serait pas un déshon-

48 Ibid., L'Aurore des Canadas (29 déc. 1840 - 22 janv. 1841). 49 Ibid., 13 déc. 1840.

50 A.V. Copy-press letter-books, No. 3. Coupures de journaux. 
neur, même pour un homme dans les veines duquel il coulerait du sang des Césars, de déployer des talents aussi extraordinaires que ceux qu'il possède, il eût préféré n'en rien faire s'il eût pu s'en dispenser; mais les fonds qu'il acquiert par là, sont scrupuleusement appliqués à promouvoir ses vues bénévoles. Quoiqu'il en soit, son apparition sur le théâtre ne porte aucune atteinte à l'élévation de ses vues philosophiques. ${ }^{51}$

Toute la presse canadienne contemporaine: Le Canadien à Montréal, La Gazette de Québec, etc., s'occupe fièvreusement de ces projets sans pareils. De son côté, avec une énergie inépuisable, Vattemare réussit à rallier à sa cause les personnages influents de l'époque, entre autres: Mgr Ignace Bourget, DenisBenjamin Viger à Montréal; le maire René-Édouard Caron, G.-B. Faribault, bibliographe et bibliothécaire du Parlement à Québec. Mais c'est surtout sur la jeunesse que s'est exercé son ascendant. Une lettre, signée par "Votre humble admirateur, Joseph Cauchon, secrétaire", provient du secrétaire de l'Assemblée générale de la Jeunesse de Québec, tenue dans la salle des séances, au Palais du Parlement, le 26 février 1841; Cauchon lui envoie une copie des résolutions adoptées lors de cette Assemblée:

S'il ne nous est pas permis, à nous qui sommes jeunes de mêler nos voix à celles des illustrations de l'Europe et du nouveau continent, pour payer comme elles le contingent de notre admiration, nous pouvons au moins vous accompagner des souhaits les plus sincères et les plus purs ...52

L'autre lettre porte la signature d'A.-N. Morin (le futur député), qui demande à Vattemare au nom des jeunes gens de Québec de retarder d'un jour son départ afin de pouvoir poser pour un artiste canadien. ${ }^{53} \mathrm{Il}$ serait intéressant de s'assurer si ce portrait n'a jamais été exécuté. 1840).

51 Ibid., Montreal Herald (26 déc. 1840); L'Aurore des Canadas (déc.

52 Ibid., Lettre de Joseph Cauchon, Québec, févr. 1841.

53 Ibid., Lettre de A.-N. Morin, Québec, 3 mars 1841. 
Reste à savoir si les résultats obtenus par Vattemare sont à la mesure de l'effervescence suscitée par sa visite. En dehors de la presse contemporaine, quelques ouvrages mentionnent sa tentative de fonder une bibliothèque publique, entre autres ceux d'Aegidius Fauteux ${ }^{54}$ et d'Antonio Drolet ${ }^{55}$. Tout récemment Claude Galarneau vient de publier un article remarquablement documenté sous le titre du "Philanthrope Vattemare" qui met au point bien des questions restées en suspens jusqu'à présent.58

En effet, il est étonnant de constater que les projets de Vattemare, bien que sanctionnés par la loi, n'aient jamais été exécutés. Car, après avoir suivi les péripéties de la visite, les assemblées et pétitions minutieusement décrites dans la presse contemporaine, il appert que le Conseil de la Ville de Montréal a présenté au gouverneur général un mémoire pour obtenir l'aide financière nécessaire à la construction de l'Institut Vattemare. Lord Sydenham accepte la demande et le 6 février un Conseil spécial promulgue une Ordonnance pour autoriser et pour mettre la Corporation de la Cité de Montréal en état d'ériger un Édifice public dans la dite Cité, pour certains objets ${ }^{57}$. La loi autorise la ville à emprunter 50,000 livres pour le projet de M. Alexandre Vattemare qui réunira les sociétés et institutions déjà existantes de Montréal, francophones et anglophones, sous l'appellation de l'Institut de Littérature, des Sciences et des Arts à Montréal. Le nouvel édifice monumental contiendra, entre autres, une bibliothèque publique, un musée, un cabinet d'histoire naturelle et une grande salle pour les réunions publiques.

${ }^{54}$ Aegidius Fauteux, "Les bibliothèques canadiennes", La Revue can. (1916), 205-214. 97-100.

55 Antonio Drolet, Les bibliothèques canadiennes (Ottawa, 1965),

56 Claude Galarneau, Le philanthrope Vattemare, dans W.L. Morton, ed., The Shield of Achilles - Le Bouclier d'Achille (Toronto, 1968), 94.

57 Ibid., 100: Statuts du Canada, t. 14. Ordonnances faites et passées par son Exc. le Gouv. Gén. et le Conseil Spécial du Bas-Canada, vol. 6, Québec. John Charlton Fisher et William Kemble, imprimeurs de la reine (1841), 495-509.

La même loi est citée dans l'ouvrage cité par Aegidius Fauteux, p. 205, dans la source suivante: Table analytique des Status, publ. en 1857 par M. Wicksteed, 4 Vict. Chap. 27. 
Le séjour montréalais de Vattemare se termine dans l'apothéose de la grande assemblée tenue le 22 janvier à l'hôtel de John Donegani, rue Saint-François-Xavier, sous la présidence de Charles Mondelet. En raison de son pouvoir magnétique, Vattemare semble avoir été capable d'aplanir les dissensions entre les deux races, car ayant annoncé les "neuf hourras" donnés par les Canadiens anglais de Montréal à leurs concitoyens canadiensfrançais, lors d'une réunion précédente, l'assemblée française s'empresse de rendre aux Anglais la pareille. Finalement l'Assemblée vote des remercîments à Vattemare, au Conseil de ville, au gouverneur général et propose qu'une messe solennelle soit chantée à l'église paroissiale. Ainsi, c'est dans une atmosphère d'action de grâces que se termine la visite de Vattemare à Montréal.

Å Québec, les événements suivent un cours presque identique. Cependant, Vattemare, avec la participation active de la Presse, ayant demandé la fusion des différentes sociétés pour former son Institut, celles-ci firent preuve d'une certaine rétivité, surtout la Société Littéraire et Historique de Québec qui détenait une charte de la Chambre d'Assemblée. Inquiété par ces tergiversations, Aubin, le rédacteur du Fantasque, prend l'initiative de suggérer que l'ancien collège des Jésuites soit affecté aux fins de l'Institut Vattemare. Ce projet soulève l'enthousiasme de l'opinion publique, les autorités militaires ayant depuis 1759 converti ce bâtiment en caserne pour y loger leurs troupes.

Plusieurs assemblées furent convoquées en vue de la promotion du projet. On y retrouve l'élite des intellectuels canadiensfrançais: les journalistes Joseph Cauchon, Etienne Parent, N. Aubin; les peintres Antoine Plamondon et Théophile Hamel. À la dernière assemblée, présidée par le maire Caron, Vattemare fait un discours éloquent sur les vingt-cinq années d'efforts déployés en Europe au service de son Système d'échanges. ${ }^{58}$

Il s'agirait maintenant de trouver le fin mot de cette étrange aventure qui avait commencé comme une marche triomphale

58 Gazette de Québec, 4 mars 1841. 
pour se terminer, partiellement du moins, dans des archives poussiéreuses. Jusqu'à présent les commentateurs ont regardé le voyage de Vattemare comme un échec total. Cependant, d'après les recherches faites dans diverses bibliothèques et archives, nous avons pu constater qu'au moins l'un de ses projets s'est réalisé: le Système d'échanges, qui a fonctionné pendant quelques années, comme nous allons le montrer par la suite.

Tout en mentionnant les côtés positifs de la visite, Galarneau attribue l'échec de la fondation de l'Institut d'une part à l'apathie de la population, d'autre part à la rivalité des intérêts institutionnels des petites sociétés. ${ }^{59}$ Par ailleurs, on ne pourrait assez insister sur l'importance du contexte politique dans cette affaire. Nous avons déjà signalé un texte paru en France qui souligne les avantages du climat politique dont a bénéficié le voyage de Vattemare. Galarneau, de son côté, élabore sur ce facteur en analysant la crise politique qui suivit la proclamation du régime de l'Union des Canadas.

N'oublions pas, cependant, qu'à l'encontre de ses actes politiques, Sydenham avait, par ses sympathies personnelles, été le grand protecteur des projets de Vattemare. Sa mort prématurée, survenue quelques mois après dans un accident de cheval, a dû beaucoup contribuer à l'avortement des projets. Du moins c'est cette raison qu'a invoquée G.-B. Faribault, dans une lettre écrite en 1847, en réponse à Vattemare qui semblait s'inquiéter du sort de son fameux Institut: "Le projet a été enterré avec Lord Sydenham."

\section{II - FARIBAULT}

\section{1 - Relations entre G.-B. Faribault et Vattemare}

L'objet de la deuxième partie de cet article est de faire connaître, d'après des documents en majeure partie inédits, les répercussions du séjour de Vattemare au Canada.

Bien que ces résultats n'aient pas l'ampleur des projets qu'il avait exposés, il n'en reste pas moins que des relations se sont

${ }^{59} \mathrm{Cl}$. Galarneau, op. cit., 106. 
établies entre la France et le Canada, d'abord dans le cadre du Système d'échanges international, par l'entremise de Vattemare, puis par celle d'autres agents.

Le premier contact a été amorcé par les relations entre Vattemare et Georges-Barthélemy Faribault, bibliographe et secrétaire-adjoint de la bibliothèque de l'Assemblée législative du Canada. La Bibliothèque publique de New York, parmi les myriades de lettres contenues dans les archives de Vattemare, en conserve trente-cinq désignées sous l'en-tête de "Lettres du Canada" dont vingt-quatre proviennent de Faribault et onze autres de divers notables canadiens. ${ }^{60}$

Les lettres de Faribault s'échelonnent de mars 1841 à novembre 1847. Il s'agit dans chacune d'envois de livres "en conformité aux ordres donnés par son Excellence le Lord Sydenham, Gouverneur Général du Canada", comme il le dit dans le style officiel de sa première lettre, ainsi que de titres demandés en échange. Donc, il ne fait pas de doute qu'un système d'échanges assez régulier ait existé entre eux pendant plusieurs années. Le mode d'envoi est également spécifié: Faribault fait d'abord parvenir les caisses à Londres à son ami George Pemberton, à l'adresse de Messrs William Pemberton Cie qui, par la suite, les expédie à $M$. Alexandre Vattemare, rue de Clichy No 48, aux soins de M. le Baron de Lagatinerie, chef de service de la Marine au Havre.

Les envois sont accompagnés de listes d'une exactitude méticuleuse, dignes de l'excellent bibliographe qu'était Faribault. La liste du premier envoi porte le titre de:

Liste des documents imprimés tirés des Archives de la Chambre d'Assemblée du Bas-Canada pour être présentés aux honorables Membres de la Chambre des Pairs et aux honorables membres de la chambre des Députés.

Puis suivent les en-têtes des divers groupes de documents :

${ }^{60}$ A.V. Copy-press letter-books, No. 3. 
Lois, statuts, etc.

Journaux ou Procès-verbaux des deux Corps législatifs du Bas-Canada (sur une liste de cinq pages) ${ }^{61}$

Quant aux envois de Vattemare nous ne pouvons nous baser que sur les titres suggérés par Faribault pour les ouvrages demandés en échange. Malheureusement, de nos jours, la vérification de ces volumes est presque impossible en raison des incendies qui ont détruit la collection du Parlement, complètement en 1849 à Montréal et partiellement en 1854 à Québec. Malgré tout, il serait intéressant de faire des recherches dans les bibliothèques canadiennes à cet égard. L'identification des volumes ne poserait pas de problèmes, par le fait que tout ouvrage ayant passé par le Système d'échanges porte sur sa page titre le timbre du Système d'échanges international, avec, au milieu, la signature d'A. Vattemare. ${ }^{62}$

Sur le plan humain, le ton de cette correspondance montre une fluctuation curieuse. De strictement officiel il devient de plus en plus amical, les en-têtes et les salutations finales de plus en plus chaleureuses. En général, les relations suivent une courbe ascendante, pour se rompre subitement avec l'intervention d'une tierce personne, le littérateur Adolphe de Puibusque, dont il sera question par la suite.

En dehors des renseignements relatifs aux documents, on trouve des notes plus personnelles dont quelques-unes ont trait aux événements historiques de l'époque. Dans l'une des lettres, par exemple, Faribault s'excuse du retard de son envoi causé par l'arrivée à Québec de sir Charles Bagot, le nouveau gouverneur qui, faute de château, a dû être logé dans le Palais législatif. Par conséquent, les archives ont été transportées pêle-mêle à Kingston d'où elles ne furent ramenées qu'après plusieurs mois. ${ }^{63}$

61 Ibid., G.-B. Faribault, lettre à A.V., Québec, 3 mars 1841.

62 Z. Haraszti, "A.V.", More Books (1927), 261. Fac-similé de la page-titre d'un livre offert par la Ville de Paris à la Ville de Boston, portant le timbre du Système d'échange international.

63 A.V., Copy-press letter-books, No. 3, Lettre de G.-B. Faribault à A.V., Québec, 11 janv. 1845. 
$\AA$ une autre reprise, en 1845 , ce sont "deux terribles incendies que nous avons essuyés le 28 mai et 23 juin derniers", qui "tout en ruinant un bon tiers de Québec ont jeté le désordre et la confusion partout", causant ainsi le retard de l'envoi.64

Dans une autre lettre, il annonce dans un post-scriptum n'ayant aucun rapport avec la lettre même, le retour au Canada de M. Papineau: "On ignore encore son dessein et ses projets. Aucune manifestation n'a eu lieu à son arrivée en Canada. ${ }^{65}$ "

Il serait curieux de découvrir pourquoi Faribault a ajouté cette note. Aurait-il eu connaissance de relations entre Vattemare et Papineau ? En somme, ils auraient pu se rencontrer à Paris en 1838, ou après le retour de Vattemare en France, entre 1841 et 1845. Hypothèse assez plausible, si l'on songe que Papineau a travaillé pendant son séjour à Paris dans les diverses archives où Vattemare devait avoir ses entrées. Par contre, les accointances libérales de Papineau n'ont dû guère le rapprocher du fidèle royaliste qu'était Vattemare. ${ }^{66}$

Reste à déterminer quelle a été la cause du refroidissement entre les deux correspondants. Notons que dès le début, après la première lettre de mars 1841 , il y a eu une interruption de quatre ans. Cependant, la seconde lettre, datée de 1845, reprend les relations sans aucune excuse, ce qui semble indiquer que cette lacune serait plutôt causée par un manque de documents que par une rupture éventuelle. D'ailleurs, c'est dans cette lettre que Faribault attribue le retard de son envoi à la visite de sir Charles Bagot; donc l'idée d'échanges réguliers est sousentendue. ${ }^{67}$

Cette continuité des envois est encore plus évidente dans l'indication ajoutée à l'adresse d'une autre lettre:

64 Ibid., Lettre de G.-B. Far. à A.V., 1er oct. 1845.

65 Ibid., Lettre de G.-B. Far. à A.V., 27 sept. 1845.

66 Bibliothèque Historique de la Ville de Paris. Ms. Lettre autographe d'A.V. à la duchesse d'Orléans, dans laquelle il invite cette dernière à l'Exposition de sa collection de dessins internationaux, mise à la disposition d'une œuvre de charité.

67 A.V. Copy-press letter-books, No. 3, Lettre de G.-B. Far. à A.V., Montréal, 11 janv. 1845. 
Avec une caisse de livres envoyés en échange

A M. Alexandre Vattemare rue de Clichy No 48 etc. $^{.8}$

Ainsi, de part et d'autre, on se plaint et s'excuse de retards. N'oublions pas que ce sont les années pendant lesquelles Vattemare est en train de raffermir ses liens avec plusieurs états des Etats-Unis dont il était devenu l'agent, et que, même aidé par son fils et son gendre, il ne pouvait plus suffire aux exigences grandissantes de son agence. Aussi ses obligations canadiennes ne devaient-elles être remplies qu'avec un certain retard. Faribault n'avait de cesse de le sommer de lui répondre:

Soyez bien persuadé, mon cher et estimable $\mathrm{Mr}$. Vattemare, que mon anxiété est des plus vives de savoir de vos nouvelles quant à tout ce qui vous intéresse et d'apprendre ce que vous avez pu réaliser pour notre Canada que vous n'avez pas oublié, j'en suis certain, parce que vous nous avez donné trop de preuves de votre amitié et de votre affection pour le Pays et ses habitants. Quand ce ne serait que quelques lignes de votre part - j'en serai satisfait. ${ }^{99}$

Rien de surprenant que, de son côté, Faribault se soit adressé à d'autres agents, comme en témoigne dès 1843 cette réponse adressée à Pierre Margry ${ }^{70}$ de la part du Conseil de la Société Littéraire et Historique de Québec:

Le Conseil de la Société Littéraire et Historique de Québec a reçu tout récemment et avec un bien vif intérêt votre lettre en date du 15 novembre dernier. Par cette lettre vous offrez obligemment de l'aider en lui procurant des copies de divers Documents relatifs à l'Histoire ancienne du Canada qui pa-

${ }_{68}$ Ibid., Lettre de G.-B. Far. à A.V., 27 sept. 1845.

69 Ibid., Lettre de G.-B. Far. à A.V., 7 juillet 1846.

70 Pierre Athanaz Margry, employé des Archives du Ministère de la marine, dont il devint, par la suite, le directeur. Il commença sa carrière d'historien comme secrétaire de l'ambassadeur américain, Lewis Cass, puis devint l'agent du gouvernement américain et canadien pour lesquels il fournissait des copies de documents. Plus tard, il écrivit quelques ouvrages concernant les activités des premiers explorateurs français en Amérique du Nord. Voir: Putney Beers, The French in North America (Baton Rouge, Louisiana State University Press [1957]), 102-103. 
raissent être déposés tant dans les Archives du Département de la Guerre, que dans celles du Bureau de la Marine, à Paris, et d'en faire la publication sous nos auspices, soit en écrivant une histoire du Canada fondée sur ces documents, ou en donnant une continuation de l'histoire de la Colonie écrite par Charlevoix. ${ }^{71}$

Les relations entre Faribault et Margry dureront jusqu'aux années 1850, mais elles seront l'objet d'une autre étude. De toute manière, ce qui est incontestable, c'est que des infidélités se sont produites de part et d'autre.

Néanmoins, dès que Faribault a vent des projets du second voyage de Vattemare en Amérique, il s'évertue à le rappeler au Canada. Il ne manque jamais d'insister sur les avantages du climat politique pour une visite éventuelle de son correspondant. En juillet 1847 il écrit de Montréal:

Le moment actuel, mon cher Monsieur, aurait été particulièrement favorable pour votre visite au Canada, car notre Législature étant maintenant en Session, vous auriez fait la connaissance des notabilités de toutes les parties du Haut et Bas-Canada, qui se trouvent réunies ici dans le moment actuel, et tous auraient été les témoins des généreux sentiments qui vous assurent la faveur du Pays, et des efforts que vous avez faits pour nous procurer une part des richesses intellectuelles de notre ancienne mère-patrie.

Je regrette donc bien vivement qu'une aussi belle occasion va se trouver perdue pour vous et pour nous, car notre Session tire à sa fin... A moins donc, que vos engagements à New York ne vous permissent de profiter du temps que durera notre Parlement pour descendre à Montréal. ${ }^{72}$

71 Bibliothèque Nationale du Québec, Département des Documents spéciaux. Lettre non signée, incomplète ( 2 pages), attribuée à Faribault par le fait qu'elle se trouve avec d'autres documents le concernant. Ajoutons que la lettre est écrite au nom du Conseil de la Société Littéraire et Historique de Québec dont Faribault était l'archiviste.

72 A.V. Copy-press letter-books, No. 3, Lettre de G.-B. Far. d̀ A.V., Montréal, 10 juil. 1847. 
Sans doute, les bruits du retour de Vattemare aux EtatsUnis avaient-ils eu l'effet de ranimer au Canada l'enthousiasme suscité par son premier séjour. Une lettre de J. Huston, bibliothécaire de l'Institut canadien, est un témoignage éloquent et, en même temps réaliste, des sentiments d'un certain groupe de la population.

$\mathrm{Au}$ début de sa lettre, il mentionne qu'il avait eu l'occasion en 1841 de manifester son approbation à "l'admirable système d'échange" de Vattemare lors de la rencontre du comité des jeunes de Québec:

J'entretiens le doux espoir de vous revoir dans le Canada au milieu de ceux que vous appeliez autrefois les Français d'Amérique. La jeunesse canadienne, soyez-en certain, vous attend avec impatience, et elle vous montrera que si les hommes mûrs n'ont pas accompli ce qu'ils vous avaient promis, elle a su garder un souvenir inéfaçable de votre passage en 1841 qu'elle veut perpétuer à Montréal en créant une institution littéraire d'après les moyens que vous lui suggériez alors.

Les bruits se sont répandus dans Montréal qu'au milieu de la riche collection de livres que vous avez apportée de France pour établir votre philanthropique système d'échange... sur des bases solides et durables, il s'en trouvait un certain nombre destiné au Canada. Mais comme malheureusement on n'a jamais mis en vigueur l'ordonnance du Conseil spécial promulgué à votre demande, pour établir un Institut par lequel les échanges du Canada se seraient faits avec les autres pays, la jeunesse franco-canadienne de Montréal craint beaucoup que cette circonstance n'ait l'effet de priver notre ville de la part qui pourrait lui revenir des richesses intellectuelles dont vous êtes le porteur. ${ }^{73}$

Certes, la conclusion de la lettre semble donner une explication plausible de l'attitude de Vattemare à l'égard du Canada. Cependant l'opinion publique était partagée et lorsqu'il devint évident que Vattemare n'avait pas l'intention de revisiter le pays,

73 Ibid., Lettre de J. Huston à A.V., Montréal, 30 sept. 1847. 
des rumeurs commencèrent à circuler à son sujet. Il est vrai que ces insinuations n'ont pas toujours été impartiales. C'est ici que se situe le rôle d'Adolphe de Puibusque, littérateur français distingué qui, profitant du mécontentement de certains cercles à l'égard de Vattemare, réussit à le remplacer. Par la suite, il sera question avec plus de détails de cet homme de lettres et bibliophile; pour le moment nous nous contenterons de citer quelques passages caustiques de l'une de ses lettres adressées à Faribault:

Tout vous a été dit... au sujet de $M$. Alexandre Vattemare; je n'ajouterai qu'un mot, c'est qu'il est l'agent de l'Amérique et non de la France, il nous est revenu du nouveau monde tout chargé de livres, de lettres et même de bills. Nous n'avons pas eu à rechercher par quels moyens il les avait obtenus, ${ }^{74}$ cela n'était pas notre affaire, mais nous avons rendu courtoisies par courtoisies, cadeaux pour cadeaux ...

$\mathrm{Au}$ fond un système d'échange universel bien réglé et gratuit serait d'une utilité incontestable; mais la profession connue de $\mathrm{M}$. Alexandre ne lui permettait pas d'imprimer à la mission qu'il s'est donné le caractère grave et digne qu'elle aurait dû avoir; il n'a pas même su renoncer au bénéfice de ses grimaces puisqu'il vous a donné des représentations payantes. ${ }^{75}$ Maintenant, un seul point vous intéresse, c'est de savoir comment vous obtiendrez la remise des divers objets qu'il a recueillis en France pour les collections du Canada. M. Quesnel pense que vous feriez bien de vous transporter à New-York et de prendre le corsaire à l'abordage. Moi, je crois avec M. Jacques Viger qu'il faut commencer par écrire...

Ici suit un long paragraphe à la fin duquel Puibusque laisse entendre à Faribault que le contenu en avait été décidé avec Jacques Viger. Toutefois, il termine sa lettre s'excusant "de la liberté que j'ai prise de vous faire parler et même écrire selon mes idées" ${ }^{76}$.

${ }^{74} \mathrm{~L}$ 'auteur de la lettre semble ignorer le pouvoir magique que Vattemare avait toujours su exercer sur les foules.

${ }^{75}$ Les recettes des représentations, du moins au Canada, étaient destinées à la promotion de l'Institut.

76 Adolphe de Puibusque, Lettre à Faribault, Montréal, 5 oct. 1847. 
Néanmoins, Faribault, après un préambule fort aimable, déplore l'impossibilité d'un voyage de Vattemare pendant la dernière session du Parlement. Puis il ajoute mot à mot le texte de la lettre précédente de Puibusque:

Outre ce que vous m'annoncez dans votre lettre j'ai appris par la voie publique que vous aviez exhibé à New-York de véritables trésors historiques, littéraires, artistiques. - Tout cela je le sais a une destination, puisque vous ne l'avez reçu que pour le remettre, mais sans porter envie aux lots de nos voisins quels qu'ils puissent être, je désire ardemment savoir quel est le nôtre. N'oubliez pas que nous n'avons été ni les moins empressés à entrer dans le système d'échange dont vous vous êtes fait le promoteur, ni les moins confiants dans les avances faites à l'Europe. On attend aujourd'hui avec quelque anxiété la distribution promise et je ne vous dissimulerai pas que le Canada a des prétentions qui le rendront exigeant. S'il vous est impossible d'apporter vous-même les objets dont vous êtes chargé pour notre parlement, veuillez me les adresser...

On n'a pas réalisé la fondation d'un institut de Montréal; le projet a été enterré avec Lord Sydenham, mais il n'est pas mort tout entier. Au lieu d'une fondation aux frais de la province, nous avons un établissement libre; c'est l'Institut canadien. Sa bibliothèque formée par des volontaires est déjà considérable, et les dons de la France pourront lui faire grand bien en fécondant le germe de son développement. En plaidant sa cause je défendrai aussi les intérêts de la Société littéraire de Québec qui a des titres plus anciens et non moins puissans ... ${ }^{77}$

Pour évaluer cette lettre il s'agit d'en relever deux points: l'insinuation selon laquelle Vattemare aurait eu l'intention de passer aux États-Unis des documents destinés par les autorités françaises au Canada et la réponse donnée par Faribault au sujet de l'échec du projet de l'Institut qui pourtant était passé en loi.

${ }^{77}$ A.V. Copy-press letter-books, No. 3, Lettre de G.-B. Far. à A.V., 10 oct. 1847 . 
En raison d'une documentation insuffisante, il est très difficile de trancher la première question. Comme il a été dit précédemment, nous n'avons pas connaissance des listes des envois faits par Vattemare à Faribault; quant aux ouvrages mêmes, ils ont dû être détruits en 1849 lors de l'incendie de l'édifice du Parlement à Montréal où la collection entière a péri. D'autre part, la correspondance que nous venons d'analyser nous a montré que Faribault fait plusieurs fois allusion aux envois de Vattemare. A partir de 1845, il accuse réception de livres en signant de "votre bien dévoué et reconnaissant serviteur". Il s'agirait donc de faire le bilan des envois pour s'assurer si Faribault avait reçu jusqu'en 1847 tout ce que Vattemare lui avait promis d'envoyer. Ceci étant malheureusement pas possible sans la connaissance des lettres envoyées par Vattemare à Faribault, les insinuations de Puibusque doivent être acceptées avec une certaine réserve.

S'il s'était agi de manquements graves, Puibusque n'aurait pas hésité à en faire un rapport au Ministère de l'instruction publique dont il avait reçu entre-temps une mission officielle. En effet, deux ans plus tard, il s'adressera au Ministère, dans une affaire de cession de livres au Parlement canadien, qu'il revendiquait au même titre que Vattemare. Comme nous le verrons par la suite, le ministre, M. de Falloux, gardera un silence prudent sur le nœud de l'affaire en essayant de satisfaire les deux partis. ${ }^{78}$

Il est incontestable que l'échec de l'Institut au Canada, du moins dans sa conception originale, a dû grandement contribuer à la décision de Vattemare de se concentrer sur les ÉtatsUnis où ses résultats étaient plus satisfaisants.

Ainsi, peu à peu, les liens se relâchent entre les deux correspondants. Nous avons déjà vu que Faribault était entré en relation avec Pierre Margry. Par ailleurs, dans deux de ses

${ }^{78}$ Ministère de l'instruction publique, France. Dossier F 172878 , Dossier Puibusque. Lettre du ministre, M. de Falloux à M. de Puibusque, 18 mai 1849. 
lettres précédentes il avait mentionné lui-même à Vattemare qu'il s'était adressé au conservateur de la Bibliothèque Royale (la Bibliothèque Nationale d'aujourd'hui) au sujet d'un échange de 15 volumes d'ouvrages modernes sur l'histoire du Canada contre un double des "Relations des Jésuites sur les Missions de la Nouvelle-France ... depuis 1632 à 1673", mais la bibliothèque ne disposait pas d'un double complet. ${ }^{79}$

Après tant d'incidents troublants, il n'est pas étonnant que, dans la dernière lettre de cette correspondance, il soit encore question de rivalités entre agents. Faribault y présente ses excuses au sujet des accusations de Vattemare qui lui reproche de s'être adressé à un agent nommé Stevens. Le ton de la lettre, écrite seulement un mois après celle de Puibusque, est très aimable et semble ne pas vouloir rompre les relations:

Je viens de recevoir à l'instant même votre lettre du 14 de ce mois et je m'empresse d'y répondre sans délai, car j'ai beaucoup à cœur de réfuter sur le champ la fausseté de la singulière histoire qui vous a été faite des livres achetés par moi d'un Mr. Stevens que je ne connais pas, et avec lequel je n'ai jamais eu de relations quelconques. ${ }^{80}$

Ainsi se termine la correspondance de Faribault avec Vattemare, selon les documents actuellement à notre disposition. Toutefois, les relations culturelles franco-canadiennes continueront, entre Faribault et Puibusque, dans une longue et harmonieuse collaboration qui durera plus de dix ans et sera très fructueuse tant sur le plan professionnel que sur le plan amical.

Il n'en reste pas moins que la source de ces nouvelles relations se trouve dans le fameux Système d'échanges d'Alexandre Vattemare.

\section{2 - Relations entre Faribault et Adolphe de Puibusque}

Le nouveau personnage qui entre en scène en 1846, AdolpheLouis de Puibusque, mérite qu'on lui consacre plus d'attention.

79 Lettre présumée de G.-B. Faribault. Voir note 71.

80 A.V. Copy-press letter-books, No. 3, Lettre de G.-B. Far. à A.V., 26 nov. 1847. 
D'une certaine notoriété à son époque, oublié aujourd'hui, il a laissé quelques ouvrages littéraires outre le souvenir de ses activités de bibliophile.

Fils du vicomte de Puibusque, administrateur militaire et écrivain, Adolphe-Louis de Puibusque naquit à Paris en 1801 et mourut dans la même ville en 1863. Il se fit recevoir avocat et fut pendant la Restauration quelque temps sous-préfet de Cherbourg. Par la suite, il s'adonna à la littérature et fit paraître de nombreux articles dans le Plutarque français, la Revue du Midi, le Recueil des jeunes personnes, etc. Il a même été directeur d'une revue politique et littéraire, intitulée France et Europe, fondée par le marquis de la Rochejaquelin; cependant celle-ci cessa de paraître en moins d'un an. ${ }^{81}$

Il publia également quelques ouvrages: la Mort de Léonard de Vinci, poème récompensé par la médaille d'or de l'Académie des Jeux floraux de Toulouse; un Dictionnaire municipal et finalement son œuvre maîtresse qui lui valut le prix de l'Académie française ainsi qu'une décoration espagnole: l'Histoire comparée des littératures espagnole et française.

Il arrive au Canada en 1846 pour le règlement d'une succession concernant la famille de sa femme et y reste environ quatre ans. Ayant joué un certain rôle dans la vie littéraire canadienne de l'époque, quelques sources contemporaines, Gagnon ${ }^{82}$ et Bibaud, publient ses données biographiques.

A ces notes vient de se joindre une biographie bien documentée dans un ouvrage récemment paru sur "Xavier Marmier et le Canada" où l'auteur, Jean Ménard, consacre plusieurs pages intéressantes à la personnalité peu connue de Puibusque. ${ }^{83}$

81 (Voir note 78). Documents concernant la fondation de la revue politique et littéraire France et Europe le 27 sept. 1838, avec A. de Puibusque comme directeur. La revue a cessé de paraître le 1er mai 1839. Ce même dossier contient également les documents relatifs à la nomination "du Lesieur de Puibusque, secrétaire général des Basses-Alpes" à la sous-préfecture de Cherbourg, le 4 avril 1830.

82 Philéas Gagnon, Essai de bibliographie canadienne (Québec, Imprimé par l'Auteur, 1895), 1: 633.

83 Jean Ménard, Xavier Marmier et le Canada (Québec, Presses de l'Un. Laval, 1967). 
Le paragraphe concernant Puibusque dans le dictionnaire de Bibaud contient les faits essentiels:

Célèbre littérateur contemporain qui a fait connaître la littérature espagnole en France et qui a été créé commandeur de l'ordre Charles III par la reine Isabelle. - Ayant épousé une Anglo-Canadienne ${ }^{84}$ qui possède de vastes terres en ce pays, il a résidé trois ans à Montréal et à Québec, a agi comme commissaire honoraire de l'Exposition à Paris et travaillé à la nomination d'un consul général de France en Canada. Quelques écrits détachés sur ce pays qu'il affectionne sincèrement, sont sortis de sa plume. ${ }^{85}$

Il nous a été possible de repérer quelques-uns des articles mentionnés dans cette note biographique, écrits d'une excellente plume et reflétant un attachement chaleureux envers le Canada:

dans le Journal de l'Instruction Publique: Le Saint-Laurent ${ }^{86}$, Le coureur de bois ${ }^{87}$, Notes d'un voyage d'hiver de Montréal à Québec; 88

dans le Journal de Québec: De la littérature française au Canada. Charles Guérin, roman de mœurs. Par monsieur Pierre Chauveau. ${ }^{89}$

Nous avons pris connaissance d'une lettre de Jacques Viger, adressée à son cousin, où il mentionne l'article de Puibusque sur

84 Jacques Viger, Lettre à Faribault, 10 févr. 1847; en parlant de Puibusque: "il est marié à une fille du colonel Taylor de la dernière guerre, et qui a été, je crois, ensuite Aide-de-camp à Québec, où il est mort".

Dans son ouvrage cité, Jean Ménard ajoute que la fille du colonel qui fut pendant plusieurs années à la tête de l'ordonnance à Québec, s'appelait Elizabeth Taylor.

85 Maximilien Bibaud, Le Panthéon canadien (Montréal, 1891), 96. Notons que le séjour de Puibusque avait duré quatre ans, d'après sa correspondance et une rectification faite par lui-même.

86 Journal de l'Instruction Publique, VI (1862), no 5 : 74-76.

87 Ibid., no 5: 90-92; no 6: 105-107.

88 Ibid., VI (1862), no 1 : $2-4$; no $2: 25-27$; no $3: 41-44$.

89 Journal de Québec. 13e année, no 99,25 août $1855,1-2$; no 101,30 août $1855,1-2$; no 102 , 1er sept. 1855 , 1. Jean Ménard, dans une note de la p. 65 de son ouvrage, donne une liste des articles ayant paru en 1861 dans une publication française intitulée: Causeries des familles. Les titres parus dans les journaux canadiens cités ci-haut figurent parmi ceux-là. 
Chauveau, paru dans l'Union du 29 juillet 1855 que le journal de Québec va reprendre en feuilleton. ${ }^{90}$

De plus, un spécimen de l'œuvre poétique de Puibusque nous est conservé au Canada par un sonnet autographe intitulé l'Illusion détruite. ${ }^{91}$

Pour en revenir à la correspondance entre Puibusque et Faribault, qui est le véritable objet de notre étude, elle s'étend sur la période de 1846 à 1857 , du moins d'après les documents qui nous sont connus actuellement. Ces lettres témoignent d'une amitié inaltérable entre les deux hommes qui se sont invités à tour de rôle à Québec, puis à Paris.

Leur premier contact s'est fait par une lettre de Puibusque, envoyée de Montréal, où cet inconnu se recommande de Vattemare qui l'avait chargé de remettre à Faribault trois brochures contenant les titres d'ouvrages destinés à la bibliothèque du Parlement:

Le nom que vous lirez au bas de cette lettre vous est sans doute entièrement inconnu, mais le titre de la brochure qui vous sera remise en même temps suffira pour vous expliquer la nature de la mission que j'ai été chargé de remplir auprès de vous. M. Wattemare (sic) s'est présenté chez moi et m'a laissé une note qui vous concerne en grande partie.

Puis suivent les titres des ouvrages que Vattemare se prépare à envoyer au Canada: un exemplaire du Mercure Français, 26 ou 28 volumes in $8^{\circ}$ au lieu des Relations des Jésuites (tant convoitées par Faribault) qu'il est très difficile de se procurer; une Histoire du Canada ou de la Nouvelle-France jusqu'en 1656 en latin, par le P. Ducreux (Francisco Cruxio), 1 vol. in-4, Cramoisy, 1664, etc. Si ces ouvrages conviennent à Faribault, Vattemare espère les lui obtenir en retour d'une des trois collections que ce dernier lui avait envoyée. Il demande que ces titres soient publiés dans la presse "afin que l'on sache que je m'occupe à remplir mes engagements envers ce pays."

90 Bibliothèque Nationale du Québec. Jacques Viger, Lettre à son cousin, Québec 25 août 1855 .

91 A.P.Q. Ms autographe, 23 août 1850. 
Après avoir transmis le message de Vattemare, Puibusque en vient aux civilités envers son correspondant inconnu. Il se plaint de la fatigue "d'une traversée de 39 jours (!) ornée d'un nombre à peu près égal de tempêtes et d'accidents de tout genre". Mais le contretemps le plus fâcheux est celui qui l'empêche de faire la connaissance de Faribault qu'il espérait trouver à Montréal, tandis que celui-ci avait dû se rendre à Québec pour l'ouverture du Parlement.

Pour finir, Puibusque fait l'éloge de l'ouvrage principal de Faribault en déclarant qu'il s'est dédommagé de l'absence de ce dernier:

en lisant d'un bout à l'autre votre catalogue raisonné et annoté d'ouvrages sur l'histoire de l'Amérique et particulièrement du Canada. Ce beau travail d'un bibliophile consciencieux m'en a plus appris en quelques pages que certaines histoires en trois ou quatre volumes. ${ }^{92}$

Faribault répond immédiatement de Québec à "l'obligeante et intéressante lettre" et ajoute dans une phrase prophétique: "j'éprouve de même un bien vif désir de faire une connaissance qui est aussi honorable pour moi qu'elle me promet d'être avantageuse dans ses résultats ce que j'espère pouvoir vous exprimer plus parfaitement de bouche sous peu." 93

Faribault aurait-il flairé au premier abord le successeur de Vattemare en Puibusque qui, n'ayant pas d'attaches avec les Etats-Unis, serait susceptible de mieux servir les intérêts du Canada ? Toujours est-il qu'il semble prendre ses précautions en ne promettant d'autres détails que de vive voix. Il termine sa lettre en annonçant l'arrivée imminente de Vattemare aux ÉtatsUnis et cite un journal de Boston selon lequel Vattemare aurait reçu une subvention de 6,000 francs pour ses frais de voyage. "Ainsi, continue Faribault, nous serions donc à la veille de revoir parmi nous cet estimable, infatigable et zélé philanthrope

92 A. de Puibusque, Lettre à Faribault, 28 déc. 1846.

93 G.-B. Faribault, Lettre à A. de Puib., 15 janv. 1847. 
qui a déjà donné tant de preuves de son affection envers les habitants du Canada. ${ }^{94 "}$

Après un intervalle de dix mois, une nouvelle lettre de Puibusque nous met en présence d'une certaine évolution. Entretemps, les deux correspondants ont fait connaissance; de plus, un troisième personnage s'est joint à eux, Jacques Viger, l'ancien maire de Montréal, "mon collègue antiquaire", comme le nomme Faribault. Ils ont déjà fait participer Puibusque aux activités qui les préoccupent dans le cadre de la Société historique et littéraire de Québec dont ils sont tous les deux fondateurs; ils s'intéressent surtout aux documents concernant Jacques Cartier.

Finalement, Puibusque fait part d'une nouvelle importante qui, de prime abord, semble pour le moins surprenante:

Je ne sais si je vous ai montré la lettre que $M$. de Salvandy, Ministre de l'Instruction publique m'a écrite le 7 février dernier et qui est ainsi conçue: J'ai l'honneur de vous inviter à faire d'actives recherches pendant votre séjour au Canada, dans les bibliothèques ou archives des documents historiques intéressant la France qui a une si grande part dans l'histoire de ce pays. J'attacherais un prix particulier à tenir de vous ces curieux documents. J'espère que toutes les autorités voudront bien vous venir en aide...

Je cite à dessein le texte même de cette lettre qui détermine nettement deux choses; l'initiative du ministre et le caractère de la mission offerte. ${ }^{95}$

Ainsi, s'appuyant sur sa mission officielle, Puibusque fait une offre formelle d'échange de documents:

Je voudrais enrichir mon pays de votre collection sauf à vous faire envoyer en échange quand je serai à Paris toutes les copies de manuscrits sur le Canada que l'on pourra trouver dans les dépôts publics. Vous m'avez déjà fait connaître ce que coûterait la copie de vos 22 ou 23 in-folio, reste à savoir exactement ce qu'ils contiennent pour faire une proposition formelle à cet égard au Ministre, si je juge après

94 Ibid.

95 A. de Puibusque, Lettre à Faribault, 5 oct. 1847. 
avoir sondé le terrain, qu'il y ait chance de succès. J'ai chargé un député de mes amis de préparer les voies en instruisant M. de Salvandy de l'heureuse correspondance qui s'est établie entre vous et le Maire de St. Malo. ${ }^{96}$

Il ne fait pas de doute que par cette lettre Puibusque empiète sur le territoire de Vattemare. Avec cela, il le fait méthodiquement, en bon administrateur, pensant aux frais et aussi aux relations nécessaires pour une telle entreprise lorsqu'il mentionne "un député de mes amis".

D'ailleurs, cette nomination qui semble lui tomber du ciel, est pour le moins dirigée. Bien que dans une lettre de 1849 , adressée au successeur de M. de Salvandy, Puibusque prétende ne pas avoir été au courant de sa propre nomination ${ }^{97}$, nous avons eu l'occasion de consulter aux Archives Nationales un dossier où l'on garde, avec la lettre précitée, trois autres lettres qui ont trait à la nomination de Puibusque.

Dans l'une des notes, écrite à la hâte, probablement par M. de Salvandy lui-même, il s'adresse à un chef de service:

Nommer immédt. M. de Puybusque, (qui est au Canada) correspdt. du M. de l'inst. pub. et l'inf. en lui demandant de profiter de son voyage dans l'intérêt du dépt. \&c.

(env. à M. le vte de Jouvenel, Député) ${ }^{98}$

Dans le même dossier, une lettre plus explicite adressée à M. le vicomte de Jouvenel, député, lui fait savoir la nomination de M. de Puibusque, en terminant par ces phrases sans équivoque:

Je suis heureux d'avoir pu prendre une décision conforme au désir que vous m'avez exprimé. Je viens d'en écrire à $M$. de Puybusque pour lui en donner avis. ${ }^{99}$

96 Ibid.

97 - F 172878 Dossier Puibusque. (Voir note 78). A. de Puibusque, Lettre à $M$. de Falloux, Ministre de l'instruction publique, 12 mars 1849 .

98 Ibid. 1 - Note s.s., s.d., probablement de la main du ministre de l'instruction publique, M. de Salvandy, adressée au Service " 2 D".

2 - Note de la même écriture, adressée au Service " $4 \mathrm{D}$ ".

99 Ibid. Lettre de M. de Salvandy au vicomte de Jouvenel, député, 24 févr. 1847. 
Sans vouloir porter atteinte à la probité de Puibusque qui, par la suite, s'est acquitté d'une manière impeccable de ses engagements envers Faribault, nous essaierons d'expliquer son comportement. Apparemment, il avait dû trouver l'idée du Système d'échanges international excellent. Il l'avait dit luimême à Vattemare, comme il le relate dans une lettre adressée à Jacques Viger:

Votre Système d'échange est une excellente chose, ai-je dit à M. Wattemare (sic), mais je ne le crois pas viable, parce qu'il ne vit que par vous, mortel comme moi. Vous avez une activité et un zèle incroyables et voyez pourtant: vous n'avez pu faire que deux voyages en sept années; vous avez 51 ans, combien de fois pourrez-vous recommencer ?... Je vois une idée, je ne vois pas d'œuvre. ${ }^{100}$

Ainsi, habile administrateur, Puibusque s'efforce-t-il à rendre le système plus "viable" en y mettant du sien, quitte à évincer l'initiateur du système. Dans sa lettre précédente adressée à Faribault, ne va-t-il pas jusqu'à annoncer son projet d'aller à Albany, puis à la Nouvelle-Orléans, explorant "en passant les divers groupes d'origine française éparpillés sur le continent américain", itinéraire de Vattemare et de plusieurs autres voyageurs français essayant de retracer les Français d'Amérique.

D'autre part, il est contestable que la confiance de Faribault en Vattemare ait été déjà ébranlée par la visite d'un certain M. de Gauchy, attaché à la Bibliothèque de la Chambre des Pairs de France qui, - Faribault le relate dans une lettre écrite à Jacques Viger - vient lui offrir de faire des échanges de documents entre les deux bibliothèques. Faribault lui ayant répondu qu'il était déjà en relation avec Vattemare, l'autre s'éleva en termes indignés contre le "charlatan" qui n'avait pas remis à la Bibliothèque de la Chambre des Pairs les documents envoyés par Faribault à son intention ${ }^{101}$. Cependant, il ne faut pas oublier que lorsqu'il s'agit de rivaux, leurs déclarations doivent toujours être jugées avec une certaine réserve. 1848).

100 A. de Puibusque, Lettre à J. Viger, s.d. (probablement de 1847 ou 101 G.-B. Faribault, Lettre à J. Viger, 2 oct. 1847. 
De toute manière, le Ministère de l'instruction publique n'a jamais voulu se prononcer dans les affaires délicates des échanges qui devenaient de plus en plus embrouillés dans la mesure où plus de personnes voulaient y participer. Deux ans plus tard, Puibusque se trouvant dans une situation analogue, décide de faire un rapport au Ministre de l'instruction publique et lui demande de prendre une décision au sujet des documents qui devaient être remis au Parlement par son intermédiaire et ceux confiés à Vattemare. ${ }^{102}$

Il fait suivre cette lettre officielle quelques mois après d'une lettre écrite par un de ses amis français de New York dont le frère travaillait au Ministère de l'instruction publique. Le signataire de la lettre demandait à son frère d'intervenir auprès du ministre, $M$. de Falloux, en faveur de M. de Puibusque et de prendre des mesures contre Vattemare et Pierre Margry qui entravaient Puibusque dans sa mission.

Il est intéressant de noter que la signature de la lettre a été raturée, probablement par le Ministère même qui devait la considérer confidentielle ${ }^{103}$. Jean Ménard ${ }^{104}$, dans son ouvrage, croit avoir identifié le signataire de la lettre en question. Il s'agirait "selon certains indices" de Henry de Courcy, littérateur français établi à New York.

La réponse du ministre ne se fit pas longtemps attendre. Avec prudence, M. de Falloux ne se prononce pas sur la question principale, sur les ouvrages que Puibusque conteste à Vattemare. Pourtant la lettre originale comporte deux listes minutieuses, dressées probablement par un employé, où figuraient séparément les ouvrages confiés à l'un et à l'autre agent. On peut cependant remarquer des ratures énergiques barrant ces précisions, tandis qu'une note, provenant probablement de la main du ministre, se contente de prendre des dispositions au sujet des ouvrages

102 - F 172878 Dossier Puibusque. Lettre d'A. de Puibusque à M. de Falloux, 12 mars 1849 (Voir note 78).

103 Ibid. Lettre confidentielle, signature raturée.

104 Jean Ménard, op. cit., 63. 
encore non expédiés qui devront être envoyés au plus tôt par l'intermédiaire du libraire désigné par Puibusque.

La lettre du ministre se termine par ces paroles chaleureuses, soulignant l'importance que la France attribue aux relations franco-canadiennes:

J'ai fait prendre une note particulière des observations que vous m'avez soumises sur la nécessité d'entretenir et de consolider les liens d'affection qui unissent la France à la population du Canada, croyez, Monsieur, que je partage à cet égard, toutes vos sympathies, et que je saisirai avec empressement toutes les occasions qui me seront offertes de contribuer à ce précieux résultat.

Je recevrai avec un vif intérêt toutes les communications que vous voudrez bien m'adresser sur les observations que vous aurez occasion de faire pendant votre... voyage; et je ne doute pas que vous n'apportiez d'utiles matériaux pour la composition de documents relatifs aux anciennes colonies françaises de l'Amérique septentrionale, qui se prépare en ce moment et doit être publié sous les auspices de mon département. 105

Cependant, opposés par le même genre d'intérêts, Vattemare et Puibusque ne cesseront guère de rivaliser dans les années à venir, ce qui ne manquera pas de laisser des traces dans la correspondance de ce dernier.

Mais pour redoutable que fût Puibusque comme rival, il s'est avéré un ami dévoué, un collaborateur fidèle et ponctuel. Il ne manque jamais, à la fin de ses lettres, de placer un mot aimable de la part de sa femme à l'intention de Madame et Mademoiselle Faribault. Lorsqu'il retourne à Paris et qu'il apprend le voyage prochain des Faribault en Europe, il s'efforce à leur trouver un logement convenable en Angleterre ${ }^{106}$, puis à Paris où il l'invite sans cérémonie: "nous entendons parler Canada tout à notre aise et chanter s'il vous plaît à la claire

\footnotetext{
105 - F 172878 Dossier Puibusque. Lettre du ministre d̀ $M$. de Puibusque, 18 mai 1849.

106 A. de Puibusque, Lettre à Faribault, 2 nov. 1851.
} 
fontaine" ${ }^{107}$. Il s'empresse d'envoyer ses félicitations à l'occasion du mariage de Mlle Georgina Faribault avec le peintre connu de Québec, Théophile Hamel ${ }^{108}$

L'amitié entre Puibusque et ses amis canadiens les plus intimes, Faribault et Viger, atteint son apogée au moment où Puibusque les propose pour être décorés de la Légion d'honneur.

Dans sa lettre adressée au Ministre de l'instruction publique, Puibusque vante les mérites de "deux savants modestes sur lesquels le regard de la France peut tomber sans exciter aucune jalousie, je veux parler de M. Jacques Viger, ancien maire de cette cité, qui passe avec raison pour le premier archéologue du Canada, et de M. G.-B. Faribault, vice-président de la Société historique et littéraire de Québec, un des bibliographes les plus laborieux de l'Amérique"... ${ }^{109}$

Quant aux affaires des échanges proprement dites qui lient Puibusque et Faribault dans les années 1847-1857, elles donnent lieu à tant de ramifications, avec la participation de tant de nouveaux personnages, que nous ne pouvons pas les traiter dans le cadre de cet article. Il suffit de noter que les transactions semblent s'être effectuées, tant sur le plan culturel que sur le plan matériel, avec tact et précision. ${ }^{110}$ Parmi leurs collaborateurs, il se passe bien quelques drames, mais on essaie d'y mettre bon ordre selon les règles de la bienséance.

En somme, les pivots de leurs relations ont été les incendies qui, ayant ravagé les bâtiments du Parlement à deux reprises, ont nécessité la reconstruction du fonds de la bibliothèque. ${ }^{111}$ C'est le premier incendie qui a donné lieu au voyage de Faribault en Europe ${ }^{112}$. La nouvelle du deuxième incendie, tout en bouleversant Puibusque, l'incite à écrire une lettre où il abonde en

107 Id., 9 nov. 1851.

108 Id., 10 nov. 1857.

109 - F 172878 Dossier Puibusque. Lettre d'A. de Puibusque à M. de Falloux, 12 mars 1849.

110 A. de Puibusque, Lettre à Faribault, 22 déc. 1853.

111 Id., 23 nov. 1849.

112 Id., 5 sept. 1850. 
conseils pratiques sur la manière de prévenir les feux par des moyens efficaces et modernes ${ }^{113}$.

Il nous reste à conclure les données esquissées dans cette étude. Dans sa carrière sans précédent, Vattemare a eu des adorateurs et des détracteurs. Cependant, ses mérites restent incontestables. Il a été l'instigateur d'un système où ses successeurs se sont évertués à le supplanter. En Europe, il avait inauguré son projet d'échanges sur un plan purement philanthropique, mais il dut chercher à se faire rembourser ses frais dans la mesure où son système prenait plus d'envergure. Il n'en reste pas moins qu'il dut souvent subvenir aux frais des échanges par ses propres moyens, au détriment de ses intérêts et de ceux de sa famille.

Puibusque, qui, en somme, a suivi ses traces, a amélioré le système quant à ses relations avec le Canada. Il eut surtout le bon sens de ne pas s'éparpiller et, en se concentrant sur ce seul pays, a été en mesure de lui rendre de réels services. Il sut habilement profiter de son état de littérateur aisé, capable de voyager à ses propres frais, ne demandant pas de rétribution pour ses services. Cela ne l'empêchait pas d'accepter des compensations, comme le faisaient d'ailleurs d'autres "dilettanti" illustres de son époque. ${ }^{114}$ Il tira également un avantage considérable de ses relations et ne manqua jamais de décocher des flèches à l'ancien métier de Vattemare, métier qui avait pourtant valu à ce dernier une réputation mondiale ainsi que l'estime des monarques et des grands esprits de son époque.

De toute manière, Vattemare a été le promoteur d'un système nouveau et, par le magnétisme de sa personnalité, par son zèle inépuisable, mérite une place d'honneur dans l'histoire des relations culturelles internationales.

ELISABETH RevaI

113 Id., 27 févr. 1854.

114 Lord Elgin, ambassadeur d'Angleterre en Turquie, père du futur gouverneur du Canada, avait acquis les célèbres statues de marbre du Parthénon, appelées depuis les "Elgin marbles", qu'il vendit par la suite, avec perte, au gouvernement anglais. 


\section{BIBLIOGRAPHIE}

Abréviations: A.N. Archives Nationales (Paris)

A.P.Q. Archives de la Province de Québec

A.S.P. Archives de la Seine et de la Ville de Paris

A.S.Q. Archives du Séminaire de Québec

B.H.V.P. Bibliothèque Historique de la Ville de Paris

B.V.M. Bibliothèque de la Ville de Montréal

B.N. Bibliothèque Nationale (Paris)

B.N.Q. Bibliothèque Nationale du Québec

D.G.I. Direction générale des Impôts (Paris)

N.Y.P.L. New York Public Library

1 - MANUSCRITS (en majeure partie documents inédits)

Georges-Barthélemy Faribault. 11 lettres adressées à Alexandre Vattemare. 1841-1847. Dans: Alexandre Vattemare, Copy-press letter books, Book No 3: Letters from Canada, N.Y.P.L. - Lettre présumée, incomplète et non signée, de la part du Conseil de la Société Littéraire et Historique de Québec, 23 janv. 1843, B.N.Q. - Calepin avec notes des livres à acheter, B.N.Q. - Lettre $\grave{a} A$. de Puibusque, 15 janv. 1847, A.P.Q. Lettres à Jacques Viger, A.S.Q.

Adolphe-Louis de Puibusque, Lettres à G. B. Faribault, 1846-1857, A.P.Q. - Lettre autographe, à un abbé canadien (probablement l'abbé Faillon), 29 juin 1856, B.V.M. - Lettres à Jacques Viger, A.S.Q. L'Illusion détruite, sonnet autographe, 23 août 1850, A.P.Q. - Archives Nationales, F161 16026 (Dossier Puibusque), A. de Puibusque, Lettre adressée au Ministre de l'instruction publique, 12 mars 1849. - Ibid., A. de Salvandy, ministre de l'instructioon publique, 2 notes (présumées, s.s.); 1 lettre signée, adressée au vicomte de Jouvenel, févr. 1847. - Ibid., Lettre s.s., adressée à un employé du Ministère de l'instruction publique par son frère, (probablement $\mathrm{H}$. de Courcy), 27 juin 1849. - Ibid., F. de Falloux, ministre de l'instruction publique, Lettre $\grave{a} A$. de Puibusque, 18 mai 1949. - Ibid., Documents concernant la fondation et la disparition de la revue France et Europe.

Romuald Trudeau, Mes tablettes, janv. 1841, B.N.Q.

Alexandre Vattemare, Copy-press letter-books, No. 3, 27 lettres adressées à Vattemare par des notables canadiens, 1841-1855, N.Y.P.L. - Lettre autographe adressée à la duchesse d'Orléans s.d., B.H.V.P. - Documents personnels, 5 actes de mariage, 3 actes de naissance, A.S.P.; Paris, Direction générale des impôts; Déclaration de succession d'A. V., 8 sept. 1864; L'écusson de la famille V. (1666), Contrat entre la Veuve V. et deux frères, 1720, B.N. - Comédies: Achille à Scyros, B.N. - Une heure à Calais, A.N. Jacques Viger, Lettre (autographe) à son cousin, Québec, 25 août 1855, B.N.Q. - Lettre à G.-B. Faribault, 10 févr. 1847, A.P.Q.

\section{2-OUVRAGES}

Henry Putney Beers, The French in North America, Baton Rouge, Louisiana State University Press [1957]. - Maximilien Bibaud, Le Panthéon Canadien, Montréal, Jos. M. Valois, 1891, p. 96. - L'abbé H. R. Casgrain, G. B. Faribault, Québec, Léger Brousseau, 1867. - Dictionary of American Biography, New York, Charles Scribner's Sons, 1916, v. 19. Antonio Drolet, Les bibliothèques canadiennes, Ottawa, Le Cercle du Livre de France, 1965. - Hugo-A. Dubuque, Alexandre Vattemare, fondateur 
de la bibliothèque de Boston, dans: Les 40 ans de la Société historique franco-américaine, 1899-1939, Boston, 1940. - Philéas Gagnon, Essai de bibliographie canadienne, Québec, Imprimé par l'Auteur, 1895, 2 v. Claude Galarneau, Le philanthrope Vattemare, le rapprochement des "Races" et des Classes au Canada, dans: W. L. Morton, The Shield of Achilles - Le bouclier d'Achille, Toronto, McLelland \& Stewart Ltd., 1968, pp. 94-108. - Henry Lyonnet, Dictionnaire des comédiens français, Paris, Librairie de l'art du théâtre, 1904, 2 vol. - Memoirs and anecdotes of Monsieur Alexandre, the celebrated dramatic ventriloquist; or The rogueries of Nicholas..., London, printed for John Lowndes, 1822. - Jean Ménard, Xavier Marmier et le Canada, Québec, Presses de l'Université Laval, 1968. - Alphonse Passier, Les échanges internationaux littéraires et scientifiques, leur histoire, leur utilité, leur fonctionnement, au Ministère de l'instruction publique de France et à l'étranger, 1832-1880, Paris, Picard, 1880. - Elizabeth M. Richards, Alexandre Vattemare and His System of International Exchanges, Columbia University, 1934, thesis. - Robert Rumilly, Papineau, Montréal, Bernard Valiquette [s.d.]. - John Sartain, Reminiscences of a very old man, 1808-1897, New York, D. Appleton \& Co., 1899. - Alexandre Vattemare, Album cosmopolite; ou Choix des collections de M. Alexandre Vattemare, composé de sujets historiques et religieux, etc., dessinés par les principaux artistes de l'Europe; accompagné de texte et fac-similé d'autographes, [Paris, Bethune \& Plon, 1837-40]. - Mason Wade, Les Canadiens français de 1760 à nos jours, Le Cercle du Livre de France [1963].

\section{3 - PÉRIODIQUES}

J. J. Ampère, "Promenade en Amérique", La Revue des Deux Mondes, Vol. 2, partie 2, 1853, p. 1007. - Bibliothèque Nationale du Québec, "3 coupures de journaux de 1847", se trouvant avec le "Calepin" de G. B. Faribault. - Bulletin des recherches historiques, vol. 3, 1897, p. 29-30; p. 73, "Le drapeau tricolore au Canada", (article d'Adolphe de Puibusque deux fois mentionné). - John Chambers, "Ventriloquism", Mirror of literature, amusement and instruction, 28: 355-356, 1836. - J.-L. Dargent, "Alexandre Vattemare, 7 nov. 1796 - 7 avr. 1864, Fondateur de l'Agence européenne des échanges", Bulletin des Bibliographies de France, 9e année, no 8 , août 1964, pp. 333-339. - Aegidius Fauteux, "Les bibliothèques canadiennes", La Revue Canadienne, Nouvelle Série, vol. XVII (1916), pp. 205214 - William Eaton Foster, "Alexandre Vattemare", Memorial biographies of the New-England historic genealogical society, v. 5: 1862-64 (1894), pp. 391-95. - Mrs. B. D. Greene, "Notes of conversation with Vattemare", Extract from Mrs. B. D. Greene's Journal, in: More Books; bulletin of the B.P.L., 2: 269-72 (nov. 1927) - Zoltan Haraszti, "The first public library", Atlantic Monthly, 194: 75-77 (July 1954). - Zoltan Haraszti, "Alexandre Vattemare", More books; bulletin of the B.P.L., 2: 257-66 (nov. 1927). - "International literary exchanges", Southern literary messenger, 9: 757 (Dec. 1843). - "Monsieur Alexandre Vattemere's [sic] System of Exchanges", Southern literary messenger, 7: 782-85 (nov. 1841). - John Pickford, "Alexandre [sic]: the ventriloquist", Notes and queries, Ser. 9: 2: 305 (Oct. 15, 1898). - Adolphe de Puibusque, "Notes d'un voyage d'hiver de Montréal à Québec", Journal de l'Instruction Publique, Vol. VI, 1862, No 1, pp. 2-4, No 2, pp. 25-27, No 3, pp. 41-44. A. de Puibusque, "Le Coureur de Bois", Journ. de l'Instr. Publ., Vol. VI, 1862, No 5, pp. 90-92, No 6, pp. 105-107. - A. de Puibusque, "Le SaintLaurent", Ibid., Vol. VI, 1862, No 5, pp. 74-76. - A. de Puibusque, "De la littérature française au Canada. Charles Guérin, roman de mours. Par 
monsieur Pierre Chauveau, (de Québec)", Journal de Québec, 13e année, no 99,25 août 1855, p. 1,2 ; no 101,30 août 1855 , p. 1-2; no 102 , 1er sept. 1855, p. 1. - Josiah Quincy, "Josiah Quincy for Vattemare's plans", More Books; bulletin of the B.P.L., 2: 267-268 (Nov. 1927). - Josiah Phillips Quincy, "Alexandre Vattemare: his services in connection with the Boston city library", Massachusetts historical society, Proceedings 1884-1885, Ser. 2: 1; 260-72 (Nov. 1884). - Elizabeth M. Richards, "Alexandre Vattemare and his system of international exchanges", Medical Library Association Bulletin, 32: 413-448 (Oct. 1844) - "Strange career of an artist", Hours at home, 7: 534-39 (Oct. 1858) - "A strange life recalled" [Alexandre Vattemare], New York Evening Post, Feb. 26, 1898. William Blake Trask, "[Biographical sketch of Alexandre Vattemare]", New England historical and genealogical register, 19: 367-69 (Oct. 1865)'. - Alexandre Vattemare, "Scrapbook from Canada", Copy-press letterbook No. 3, Coupures de journaux de 1840-1841. - Hippolyte Vattemare, "Notices of the life of Alexander [sic] Vattemare, founder of the System of international exchange", trans. from the French by Salomon Alofsen, Historical magazine, Ser. 2: 4: 297-300 (Dec. 1868). 